\title{
Allogeneic Neural Stem Cell Transplant Promotes Functional Recovery of Locomotion After Complete Transected Spinal Cord Injury Predominantly by Secreting Neurotrophic Factors
}

Shuo Liu

Nanjing University Medical School Affiliated Nanjing Drum Tower Hospital

Caixia Fan

Suzhou Institute of Nano-tech and Nano-bionics

\section{Yuanyuan Xie}

Nanjing University Medical School Affiliated Nanjing Drum Tower Hospital

Liudi Wang

Nanjing University Medical School Affiliated Nanjing Drum Tower Hospital

Yanyan Cui

Chongqing Medical

Bin Wang ( $\square$ wangbin022800@126.com)

The Affiliated Drum Tower Hospital of Nanjing University Medical School https://orcid.org/0000-00033981-8849

\section{Research}

Keywords: Spinal cord injury, Neural stem cells, Neurotrophic factors, Myelin, Paracrine

Posted Date: January 12th, 2021

DOl: https://doi.org/10.21203/rs.3.rs-142155/v1

License: (c) (1) This work is licensed under a Creative Commons Attribution 4.0 International License. Read Full License 
1 Allogeneic neural stem cell transplant promotes functional recovery of

2 locomotion after complete transected spinal cord injury predominantly by

3 secreting neurotrophic factors

4 Shuo Liu ${ }^{1 \#}$, Caixia Fan ${ }^{2 \#}$, Yuanyuan $\mathrm{Xie}^{1}$, Liudi Wang ${ }^{1}$, Yanyan $\mathrm{Cui}^{3^{*}}$, Bin Wang ${ }^{1 *}$

$5 \quad{ }^{\#}$ Contributed equally to this work.

$6{ }^{1}$ Clinical Stem Cell Center, the Affiliated Drum Tower Hospital of Nanjing University Medical

$7 \quad$ School, Nanjing 210000, P. R. China

$8{ }^{2}$ Key Laboratory for Nano-Bio Interface Research, Division of Nanobiomedicine, Suzhou

9 Institute of Nano-Tech and Nano-Bionics, Chinese Academy of Sciences, 398 Ruoshui Road,

Suzhou 215123, China

${ }^{3}$ Department of Cell Biology and Genetics, Chongqing Medical University, Chongqing 400016,

China

*Corresponding author (Bin Wang, Ph.D., 321 Zhongshan Road, Nanjing 210008, China.

Telephone: 86-25-68182508; e-mail: wangbin022800@126.com, Yanyan Cui, 1 Yixueyuan Road,

\section{Abstract}

Objective: Cell-based therapy is a promising strategy for spinal cord injury (SCI) repair, but faced the challenges to direct the neuronal differentiation of appropriate neuron subtypes for achieving the neuronal replacement. We investigated whether allogeneic beforehand in vitro differentiated neural stem cells (NSCs) could relieve the adverse effects of regeneration inhibitory niche and promote motor functional recovery by accomplishing neuronal replacement after transplant into 
SCI rats.

Methods: Collagen scaffold combined with digested NSCs, NSC sphere, differentiated neurons, and sphere of differentiated neurons were transplanted into completely transected SCI in rats and therapeutic outcomes were investigated. Next, we enriched complex of neurotrophic factors secreted from culture medium of NSCs, neurons, and sphere of neurons and a total of $2 \mathrm{mg}$ total enriched protein combined with collagen scaffold were transplanted into SCI to further assay whether allogeneic NSCs transplant promotes the recovery of SCI predominantly by secreting neurotrophic factors.

Results: NSCs differentiated into neurons in density-dependent manner in vitro and sphere of NSCs could counteract myelin-induced inhibition of neuronal differentiation. Collagen scaffold combined with digested NSCs, NSC sphere, differentiated neurons, and sphere of differentiated neurons were transplanted into completely transected SCI in rats. Overall the cell treatment groups had a much better locomotor recovery, tissue remodeling, and newborn neuron formation than alone collagen scaffold treatment, compared with alone collagen material transplant and control group. However, unexpectedly, the differentiated cell treatment (differentiated neurons and sphere of differentiated neurons transplants) did not present striking better locomotor recovery than the undifferentiated NSCs and sphere of NSCs treatments, only sphere of neurons showed a slight increase in BBB score compared to other cell treatments. Next, we enriched complex of neurotrophic factors secreted from culture medium of NSCs, neurons, and sphere of neurons. BBB score analysis showed that the secreted neurotrophic factors from NSCs, neurons, and sphere of neurons would promote functional recovery of SCI to the same extent.

Conclusion: Allogeneic NSCs transplant promotes functional recovery of SCI predominantly by 
secreting neurotrophic factors, not direct neuronal replacement of differentiated neurons from transplanted cells.

Keywords: Spinal cord injury, Neural stem cells, Neurotrophic factors, Myelin, Paracrine

\section{Background}

Spinal cord injury (SCI) generally loses segmental motor and interneurons motor neurons below the lesion, causing motor and sensory functions. Until today SCI is still one of the most serious injuries of central nervous system (CNS) and SCI repair is a major problem in clinic. After SCI, a series of pathophysiological reactions not only causes a progressive loss of nerve tissue, but also formed an inhibitory niche of regeneration at injured site, which includes glia scar formation, deficiency of neurotrophic factors, inflammatory responses, and release of myelin proteins[1-3].

its self-renew potential and differentiation capability into neurons[4]. Ideally the newborn neurons differentiated from transplanted NSCs can replenish lost neurons and form functional relays connecting spinal cord segments across the lesion gap. However, in mouse SCI model, endogenous NSCs derived from central canal of spinal cord predominantly differentiated into astrocytes and oligodendrocytes at injured site, but inefficiently differentiate into neurons $[5,6]$.

Our previous study once firstly showed the regeneration inhibitory myelin protein from spinal cord could inhibit the differentiation of NSCs into neurons, but promoted their differentiation into glial cells[7]. Myelin associated proteins includes Nogo-A, MAG, and OMgp. These three inhibitory molecules can recognize and bind their common receptor $\mathrm{NgR} 1$, further recruiting $\mathrm{p} 75$, TROY and LINGO - 1 receptor and forming a complex, resulting in axon growth inhibition by 
phosphorylating RhoA and rearranging cell cytoskeleton[8]. Accumulating evidence indicate that rat NSCs cultured in vitro derived from either adult or embryonic CNS were transplanted into intact or injured adult spinal cord and exclusively differentiate into glial lineages, not neurons[9-11]. Due to regeneration inhibitory milieu after SCI, although NSC-based therapy is considered to have a great potential in SCI repair, it fails to achieve satisfactory outcomes in animal experiments and clinical trials[12-14].

neurons in vitro and then transplanted into the injured site, to avoiding the neuronal differentiation inhibition of adverse environment after SCI. The results showed that the motor function and newborn neurons was improved after transplant of primary NSC sphere, NSCs digested into single cell, differentiated cells from NSC sphere and NSCs digested to single cell compared to un-treatment group after SCI, but there was no significant difference among the transplant groups. Gene microarray revealed there were no significant differences in mRNA expressions of neurotrophic factors among NSC sphere, NSCs digested into single cell, differentiated cells from NSC sphere and NSCs digested to single cell and it was validated by qPCR, indicating it was secreting neurotrophic factors, not newly generated neurons in NSC therapy, predominantly promoting the functional recovery of locomotion after SCI. Further we enriched neurotrophic factors from culture medium from NSC sphere, differentiated cells from NSC sphere and NSCs digested to single cell, which then were transplanted with collagen scaffolds into the injured sites after SCI. The animals gained similar locomotion recovery in neurotrophic factor transplant groups. Therefore, we considered that allogeneic NSC transplant promoted functional recovery of 
locomotion after complete transected SCI predominantly by secreting neurotrophic factors from transplanted cells, not cell itself.

\section{Materials and methods}

\section{Antibodies and Reagents}

Antibodies: GFAP (Cat\# ab929, Abcam, Cambridge, UK); $\beta$-III tubulin (Cat\# ab7751, Abcam, Cambridge, UK); Nestin (Cat\# ab6142, Abcam, Cambridge, UK) ; BDNF ELISA kit (Cat\# ER012-96, ExCell Bio, China ); NGFbeta (Cat\# EK0471, BOSTER, China).

\section{NSCs Culture}

The hippocampus were dissected from ten Sprague- Dawley(SD) rat embryos (day 12-14, specific pathogen free(SPF)) and was digested in accutase at $37{ }^{\circ} \mathrm{C}$ for 20 minutes. The tissue was washed twice with PBS and then was pipetted into single cell suspension in serum-free Dulbecco's modified Eagle medium: Nutrient Mixture F-12 (DMEM/F12) medium (Gibco, Grand Island, NY, USA). After filtering with $40 \mu u m$ cell sieve, the cell suspension was cultured in 25 cm2 tissue culture flask (Corning, NY, USA) with serum-free DMEM/F12 medium containing 20 $\mathrm{ng} / \mathrm{mL}$ epidermal growth factor (EGF,Peprotech Asia), $20 \mathrm{ng} / \mathrm{mL}$ basic fibroblast growth factor (bFGF, Peprotech Asia, Rehovot, Israel), 2\% B27 (Gibco, Grand Island, NY, USA), and 1\% penicillin-streptomycin(Gibco, NY, USA). After 8-day culture, neurosphere were formed and the cells were enzymatically dissociated for following experiments.

For Immunocytochemistry, NSCs were seeded in 12 well culture plate (Corning, NY, USA) at 3

$\times 105$ cells per well. The maintaining medium was DMEM (high glucose, Gibco, Grand Island, NY, USA) with N2 supplement(Gibco, Grand Island, NY, USA). In the presence of myelin, Myelin basic protein (MBP), Glutathione S-transferase (GST), or Nogo-66 for 8-day, the terminal 
differentiated cells was assessed by immunocytochemistry. For the biochemical assays, cells were treated with factors and harvested at the time points mentioned in the figure legends.

\section{Myelin Preparation}

As previously reported, myelin was prepared from the spinal cord of adult rats[15]. To obtain myelin, tissue was homogenized in $0.3 \mathrm{M}$ sucrose and stratified under 1.23 and $0.85 \mathrm{M}$ sucrose gradients. The samples were centrifuged at $75000 \mathrm{~g}$ for $45 \mathrm{~min}$, and the crude myelin components were collected at the $0.85 / 1.23 \mathrm{~m}$ interface. Then, the crude myelin components were washed twice by osmotic shock, resuspended with $0.32 \mathrm{M}$ sucrose, layered on $0.85 \mathrm{M}$ sucrose, centrifuged and collected from $0.32 / 0.85 \mathrm{M}$ interface. The excess sucrose myelin was taken out and suspended in DMEM 1:1, homogenized, and stored at 280uC for following experiments.

\section{SCI modelling}

All animal experiments were performed in the light of Guide for the Care and Use of Laboratory Animals from National Institutes of Health. The study was approved by the Research Ethics Board of The Affiliated Drum Tower Hospital of Nanjing University Medical School.

A total of 90 adult female SD rats (200-250 g, SPF, Nanjing Medical University,) were housed in temperature and humidity-controlled animal dormitories with a light / dark cycle of 12 hours for 10 days. All rats were anesthetized with isoflurane (RWD, Shenzhen, China). The back of anesthetized rats was depilated and the skin was washed with povidone iodine solution. A $2 \mathrm{~cm}$ midline incision was made to expose the T9-T11 vertebrae. A $2 \mathrm{~cm}$ midline incision was made $\mathrm{r}$ to expose the T7-T9 vertebrae and a $3 \mathrm{~mm}$ of T8-9 spinal cord was completely transected with eye scissors. A $4 \mathrm{~mm}$ long and $2 \mathrm{~mm}$ diameter bundle of linearly ordered collagen fibers loading 106 NSCs or neurons, or sphere of NSCs or neurons containing 106 cells was transplanted into the 
131

defect of transected spinal cord and then the muscle issue and skin were sewn. All experimental rats were allocated into: control group without any treatment $(n=15)$; material group with collagen fibers implantation $(n=15)$; NSCs group with collagen fibers loading 106 NSCs $(n=15)$; sphere of NSCs group with collagen fibers loading sphere of NSCs containing 106 cells $(n=15)$; neurons group with collagen fibers loading 106 neurons $(n=15)$; and sphere of neurons group with collagen fibers loading sphere of neurons containing 106 cells $(n=15)$. After the treatment, the musculature and skin were separately sutured in layers.

To treat SCI with enriched neurotrophic factors, a total of $600 \mathrm{ug}$ enriched proteins in 200 $\mu \mathrm{H}$ 2O were dripped onto collagen scaffold, which was transplanted into injured site of each SCI rat after desiccation in laminar flow cabinet.

\section{Histological analysis}

Rats were sacrificed at 8 weeks after surgery. All rats were anesthetized with isoflurane. Rats were perfused with PBS and 4\% paraformaldehyde in PBS. Spinal cords were dissected, post-fixed 48 hours at $4{ }^{\circ} \mathrm{C}$, and transferred to $20 \%$ sucrose (48 hours at $4{ }^{\circ} \mathrm{C}$ ) and then $30 \%$ sucrose (72 hours at $4{ }^{\circ} \mathrm{C}$ ). The segments were then embedded in paraffin and cut into $10-\mu \mathrm{m}$ thick sections by Lecia RM2235 (Leica Biosystems, Wetzlar, Germany). Adjacent tissue sections were stained with hematoxylin and eosin (H\&E) for general observation of cellular and extracellular matrix features. The H\&E images were obtained using a Leica SCN400 slide scanner (Leica Microsystems, Germany).

\section{Basso-Beattie-Bresnahan (BBB) scoring}

BBB scoring was performed to assess the motor functional recovery of SCI animals. In present study, BBB scoring was carried out once per week during the observation period of 8 
153

154

weeks after SCI. If the SCI model was successful, the BBB scores of SCI rats were about 0 , which represented the complete paraplegia of hind legs immediately after surgery. We observed that in four cell transplantation groups the recovery was better than that of the control or material group during 2-8 weeks, while there was no sustained significant difference between the four cell transplantation groups. And the observation of anatomical features at week 8 after SCI was consistent with the above behavioral scores. These results demonstrated that functional recovery after sever SCI could not been effectively improved when treated with sphere of NSCs or neurons compared to single NSCs or neurons.

\section{Total transcriptome analysis}

The Affymetrix GeneChip® Rat Genome 2302.0 chip (Shanghai Biotechnology Corporation, China) was utilized to analyze the total transcriptome in rat among three groups. The samples of primary rat NSCs cultured for 7 days were taken as NSCs group (without differentiation). NSCs with enzymatic digestion into individual or not were induced to neural differentiation for 7 days in serum-free DMEM/F12 medium with B27 supplement, respectively named as Neuron group and Sphere of Neuron group. The chip contains 31,000 probe sets for 28,000 rat mRNAs derived from authoritative databases, including GenBank, dbEST, and RefSeq. TRIZOL Reagent (Cat\# 15596-018, Life technologies, Carlsbad, CA, US) was used for the extraction of total RNAs. According to the manufacturer' $s$ instructions and checked for a RIN number to inspect RNA integrity (Agilent Bioanalyzer 2100, Agilent technologies, Santa Clara, CA, US). RNeasy mini kit (Cat\# 74106, QIAGEN, GmBH, Germany) and RNase-Free DNase Set (Cat\# 79254, QIAGEN, GmBH, Germany) were used for the further purified of qualified total RNA. Total RNA was amplified, labeled and purified by using GeneChip 3' IVT PLUS Reagent Kit (Cat\# 902416, 
175

Affymetrix, Santa Clara, CA, US) and biotin labeled cRNA was obtained by the manufacturer's instructions. Array hybridization and washing was performed using GeneChip® Hybridization, Wash and Stain Kit (Cat\# 900720, Affymetrix, Santa Clara, CA, US) in Hybridization Oven 645 (Cat\# 00-0331-220V, Affymetrix, Santa Clara, CA, US) and Fluidics Station 450 (Cat\# 00-0079, Affymetrix, Santa Clara, CA, US) followed the manufacturer's instructions. Data acquisition Slides were scanned by GeneChip ${ }^{\circledR}$ Scanner 3000 (Cat\# 00-00212, Affymetrix, Santa Clara, CA, US) and Command Console Software 4.0 (Affymetrix, Santa Clara, CA, US) with default settings. Raw data were normalized by MAS 5.0 algorithm, Gene Spring Software12.6.1 (Agilent technologies, Santa Clara, CA, US). The differential expressed genes among groups were screened using $\mathrm{T}$ test analysis and the criteria were as follows: 1) 2-fold difference: fold change (linear) $=<0.5$ or fold change $($ linear $)>=2.2)$ T-test $p$-value $<0.05$ and $p$-value $<0.01$.

\section{Bioinformatics Analysis}

Principal Component Analysis (PCA) and Partial Least Squares Discrimination Analysis (PLS-DA) were completed by using the R function prcomp from the stats package with default parameters and the mixOmics package (https://CRAN.R-project.org/package= mixOmics). Pathway analysis was performed using the Kyoto Encyclopedia of Gene and Genomes (KEGG) (http://www.genome.ad.jp/kegg/mapper.html) pathway to identify significant enrichment of biochemical pathways. Hierarchical Cluster Analysis (HCA) was processed with the pheatmap package (https://CRAN.R-project.org/package=pheatmap). Functional classification and annotation were performed with Gene Ontology (GO) using the DAVID bioinformatics resources 6.8 (http://david.ncifcrf.gov/).

\section{Quantitative RT-PCR (qPCR) analysis of mRNA expressions}



quantitative reverse transcription-PCR (RT-PCR) was carried out by a two-step reaction according to the kit's instructions. For first step, RT reactions were performed in a GeneAmp ${ }^{\circledR}$ PCR System 9700 (Applied Biosystems, USA), each reaction system consisting of total RNAs (0.5 $\mu \mathrm{g})$, oligo in a total volume of $10 \mu \mathrm{l}$. The $10 \mu \mathrm{l}$ of RT reaction mix then was diluted into $100 \mu \mathrm{l}$ of cDNA volume using nuclease-free water and stored at $-20^{\circ} \mathrm{C}$ for quantitative PCR. Real-time PCR reaction mixture contained contained $1 \mu \mathrm{l}$ of cDNA template, $5 \mu 1$ of 2 X LightCycler ${ }^{\circledR} 480$ SYBR

Green I Master Mix (Roche, Swiss), $0.2 \mu 1$ of forward and reverse primer mixtures (20 uM) in a total $10 \mu$ l volume. Quantitative PCR was performed in a LightCycler® 480 II Real-time PCR

\begin{tabular}{ll}
\hline Gene & \multicolumn{1}{c}{ Primer sequences } \\
\hline GAPDH & F:5'-GAAGCTCATTTCCTGGTATGACA-3' \\
& R:5'-ATTGATGGTATTCGAGAGAAGGG-3' \\
\hline GDNF & F:5'-CGCTGACCAGTGACTCCAAT-3' \\
& R:5'-TGGTAAACCAGGCTGTCGTC-3' \\
\hline BDNF & F:5'-TACCTGGCGACAGGGAAATC-3' \\
& R:5'-GGTGGAACATTGTGGCTTTGC-3' \\
\hline NGF & F:5'-CGCATCGCTCTCCTTCACAG-3' \\
& R: 5' '-TGGCCAGGATAGAAAGCTGC-3' \\
\hline
\end{tabular}




\begin{tabular}{ll}
\hline CNTF & F:5'-GGAATCTTATGTAAAACATCAGGGC-3' \\
& R:5'-GGAGGTTCTCTTGGAGTCGC-3' \\
\hline bFGF & F:5'-GCGACCCACACGTCAAACTA-3' \\
& R:5'-TCCGTGACCGGTAAGTGTTG-3' \\
\hline VEGF & F:5'-AGAAAGCCCATGAAGTGGTG-3' \\
& R:5'-ACTCCAGGGCTTCATCATTG-3' \\
\hline HGF & F:5'-ACAGCTTTTTGCCTTCGAGC-3' \\
& R:5'-TGTCGGGATATCTTTCCGGC-3' \\
\hline IGF2 & F:5'-AAGCCTACAAAGTCAGCTCG-3' \\
& R:5'-GGTCTTGTTTCCTGCACTTC-3' \\
\hline EGF & F:5'-ACGAACTTGTTGCCTGTCCA-3' \\
& R:5'-TCGGAGAAGGAACAACCAGTG-3' \\
\hline PDGF & F:5' 'CAAGACGCGTACAGAGGTGT-3' \\
& R:5'-TCGATCTTTCTCACCTGCACC-3 \\
\hline LIF & F:5'-TCTTGGCCACAGGGATTGTG -3' \\
& R:5'-CACATGGCCCACATGGTACT-3 \\
\hline
\end{tabular}

Enrichment of neurotrophic factors from culture medium

After 7-day primary culture, NSCs formed neurospheres and treated by following three ways

to collect medium. A total of $3 \times 106$ NSCs were transferred into each new $25 \mathrm{~cm} 2$ tissue culture flask and cultured for $12 \mathrm{~h}$ with $10 \mathrm{ml}$ basal DMEM/F12 medium without any supplement. The conditioned medium was harvested to enrich neurotrophic factors from NSCs. A total of $1 \times 106$ primary NSCs enzymatically digested into single cells or not were seeded on each new $25 \mathrm{~cm} 2$ tissue culture flask with DMEM/F12 medium with 10\% FBS. After culture for $10 \mathrm{~h}$, all cells were robustly adhered and DMEM/F12 medium with B27 supplement replaced the old medium to direct the differentiation of NSCs. The differentiation medium was changed every 3 days. Until

223 day 7, the number of cells grew up into about $3 \times 106$. Then old medium was replaced with $10 \mathrm{ml}$

224 basal DMEM/F12 medium and conditioned medium was harvested after $12 \mathrm{~h}$ for enriching the

225 neurotrophic factors secreted from neurons and neurons of sphere.

Enzyme-linked immunosorbent assay (ELISA) 
supernatant according to the manufacturer's instruction (Rat NGF ELISA kit, Cat No. EK0471, Boster Biological Technology, Co.,LTD, China; Rat BDNF ELISA kit, Cat NO.ER012-96, Excell Bio Co.Ltd, China) using a plate reader at $450 \mathrm{~nm}$. The final concentration of each cytokine in medium was determined based on the standard curve.

\section{Statistical analysis}

The data were presented as Mean \pm Standard Derivation $(\mathrm{SD})$ from a minimum three replicates of each experiment. A $p<0.05$ was considered statistically significant. Graphpad prism version 5.0 (GraphPad Software, San Diego, CA, USA) was used to determine significance and generate graphs. Statistical analyses were performed using a standard one-way analysis of variance (ANOVA) with Tukey's post hoc multiple comparisons.

\section{Results}

The percentage of neuronal differentiation of NSCs in vitro was increased when more cells were seeded into a well of 24 well-plate, indicating there was a density-dependent manner in neuronal differentiation of NSCs (Figure 1A-B). The cell density of NSC sphere was very high and thus the neuronal differentiation was maximal when NSC spheres were seeded without enzyme digestion (Figure 1A-B). To view the overall perspective of NSCs' differentiation, hierarchical scan of immunostaining slides was performed by confocal. The majority of differentiated cells were GFAP positive astrocytes when enzyme digested NSCs seeded (Figure 1C). In contrast, the Tuj-1 positive neurons were predominant in differentiated cells NSC sphere (Figure 1D). Furthermore, there were many long processes of neurons connected with each other sphere (Figure 1D). With consistent previous study, myelin could inhibit the neuronal differentiation of enzyme digested NSCs, but had little effect on the neuronal differentiation of 
intact NSC sphere (Figure 1E-F).

We hypothesized that the transplant of differentiated neurons derived from NSCs in vitro could avoid the in vivo adverse niche of neuronal differentiation of NSCs after SCI and promote the motor functional recovery. The adult rats were performed complete spinal cord transection and allocated into six groups according to different treatments (Control group: without any treatment, Material group: collagen scaffold transplant, NSCs group: collagen scaffold plus primary enzyme digested NSCs, Sphere of NSCs group: collagen scaffold plus NSC sphere, Neurons group: collagen scaffold plus differentiated cells derived from enzyme digested NSCs, Sphere of neurons group: collagen scaffold plus differentiated cells derived from NSC sphere ) (Figure 2A). Collagen scaffold was used as attaching and supporting material for transplanted cells in this model. Each experimental rat was assessed the $\mathrm{BBB}$ locomotor score every week post SCI, range from 0 (complete paralysis) to 10 (healthy) (Figure 2B). The control group only had a very limit locomotor recovery and material groups had a slightly higher BBB locomotor score than control group. Overall the cell treatment groups had a better locomotor recovery. However, unexpectedly, the differentiated cell treatment (neurons and sphere of neurons groups) did not present striking better locomotor recovery than the undifferentiated cell treatments (NSCs and Sphere of NSCs groups), only sphere of neurons showed a slight increase in BBB score compared to other cell treatments (Figure 2B). After 8 weeks post-surgery, all the rats were sacrificed and the samples of injured spinal cords were harvested for further analysis. The H\&E staining showed a less tissue remodeling and huge cavity in control group and material treatment had a better tissue remodeling and smaller cavity. All the cell treatment groups had a similar outcome with tissue a good tissue remodeling in injured sites (Figure 2C). Relatively, collagen material combined sphere of neurons 
lead to the best tissue remodeling and smallest volume of cavities nearby the injured sized.

In the original experimental scenario, we anticipated the transplantation of differentiated neurons or sphere of neurons would markedly enhance the recovery of motor function after SCI in rats compared with the NSCs or sphere of NSCs faced with neuronal differentiation inhibitory niche in vivo, because their neuronal differentiation had finished in vitro, leading to more neurons in injured sites involving in nerve regeneration. However, we did not observe the expected a much better outcome of motor functional recovery in differentiated neurons or sphere of neurons groups. Tuj-1, also named neuron-specific class III beta-tubulin, is a neuronal marker of neurons. Thus Tuj-1 immunostaing was performed to investigate the newborn neurons in injured sites after SCI (Figure 3). The panoramic scanning of the horizontal sections of spinal cord showed the Tuj-1 staining was heavy and axially well-ordered outside of injured site, but was clear-cut deficit in damaged lesion (Figure 3A). In high-magnification of injured area, Tuj-1 positive cells were observed and the staining was distinct among groups with corresponding treats (Figure 3B). The positive of newborn neurons (Tuj-1+ cells) were sparse in control and material groups. In contrast, there were much more newly formed neurons observed in the four groups with different cell transplant (Figure 3B). Further quantified data showed the percentage of Tuj-1+ neurons in material group was $7.52 \pm 2.22 \%$, higher than $12.07 \pm 2.61 \%$ in control group (Figure 3C). Overall, the other four groups with cell treatment had parallel percentages of Tuj-1+ neuron, markedly higher than that in control and material groups.

\section{Transcriptome analysis}

To compare the transcriptome of neurons differentiated from single NSC (neuron group) and neurons differentiated from sphere of NSCs (sphere neuron group), the Affymetrix GeneChip® 
Rat Genome 2302.0 chip was utilized to analyze the transcription profiles among NSCs, neuron, and sphere of neuron groups. A total of 168 differentially expressed genes (with 2 folds change) were identified between neuron and sphere of neuron groups, with 144 up-regulated genes and 24 down-regulated genes, respectively. Heat map showed the differentially expressed genes from neuron group samples compared with sphere of neuron group samples (Figure 4A). Each column represents one tissue sample and each row represents one gene. The differentially expressed genes clearly self-segregated into neuron and sphere of neuron clusters. Pathway analysis of differentially expressed transcripts was performed using the Kyoto Encyclopedia of Gene and Genomes (KEGG) (http://www.genome.ad.jp/kegg/mapper.html) pathway to identify significant enrichment of biochemical pathways among neuron and sphere of neuron groups. The results showed that neuron projection development related processes were mainly enriched in neuron samples, and cell adhesion and motility, proliferation processes were mainly enriched in sphere of neuron samples (Figure 4B). There was an obvious diversity in enrichment of biochemical pathways among neuron and sphere of neuron, however, overall, we did not observe a significant difference in motor functional recovery and newly borne neurons in juried sites after SCI among four cell transplant groups. Thus, we conjectured there were some common characteristics shared in four cell transplant groups, which contribute to the recovery of SCI. The further heat map focused on neurotrophic factors showed there was a comparable mRNA expression profile among NSC, neuron, and sphere of neuron groups (Figure 5). mRNA expressions of neurotrophic factors

Then we analyzed the mRNA expressions of neurotrophic factors including VEGF, BDNF, GDNF, TGF $\alpha$, EGF, IGF2, NGF, PDGF $\alpha$, bFGF, LIF, and CTNF by qPCR. The results showed 
there was no significant difference in mRNA expressions of most examined genes including BDNF, GDNF, EGF, IGF2, NGF, PDGF $\alpha, b F G F$, and LIF among groups, except for VEGF, TGF $\alpha$, and CTNF (Figure 6A). To validate the mRNA expression results, ELISA was performed to determine the protein expressions in medium secreted by NSC, neuron and sphere of neuron using BDNF and NGF ELISA kit. The ELISA results showed the protein expression of BDNF and NGF was similar with their mRNA expressions among NSC, neuron, and sphere of neuron (Figure 6B).

\section{Complex of neurotrophic factors promoting moto functional recovery}

We enriched complex of neurotrophic factors secreted from NSCs, neurons, and sphere of neurons from corresponding culture medium. We harvested a total of 600ug enriched proteins per vial which was re-dissolved in $200 \mu \mathrm{PBS}$ to determine the concentrations of BDNF and NGF using ELISA kits. There was no significant difference in protein concentrations of NGF and BDNF among NSCs, neurons, and sphere of neurons before and after enriching (Figure 7A). But the concentrations of both NGF and BDNF in enriched medium were about 7-times higher than that in basal medium (Figure 7A). To test the role of secreted neurotrophic factors on motor functional recovery of SCI, a total of $2 \mathrm{mg}$ total enriched protein secreted from NSCs, neurons, and sphere of neurons, respectively was cross-linked with collagen scaffold and then transplanted into completely transected spinal cord at T8-10 in rat. The BBB scores were investigated every week post-surgery. The result showed BBB scores were gradually increased among all groups during 1-4 weeks post-surgery, but kept stable during the rest observed period (Figure 7B). BBB scores in NSC, neuron, and sphere of neuron medium groups were at similar level, but markedly higher than that in control and material groups, indicating the secreted neurotrophic factors from NSCs, neurons, and sphere of neurons would promote functional recovery of SCI to the same extent. 


\section{Discussion}

In this study, we found NSCs differentiated into neurons in density-dependent manner in vitro and sphere of NSCs could counteract myelin-induced inhibition of neuronal differentiation. Collagen scaffold combined with digested NSCs, NSC sphere, differentiated neurons, and sphere of differentiated neurons were transplanted into completely transected SCI in rats. Alone collagen material transplant had a slightly higher BBB locomotor score, compared with control group. Overall the cell treatment groups had a much better locomotor recovery, tissue remodeling, and newborn neuron formation than alone collagen scaffold treatment. However, unexpectedly, the differentiated cell treatment (differentiated neurons and sphere of differentiated neurons transplants) did not present striking better locomotor recovery than the undifferentiated NSCs and sphere of NSCs treatments, only sphere of neurons showed a slight increase in BBB score compared to other cell treatments. Transcriptome analysis showed that there was an obvious diversity in enrichment of biochemical pathways among neuron and sphere of neuron. However, there was no significant difference in mRNA expressions of most examined neurotrophic genes such as BDNF and GDNF among groups. Next, we enriched complex of neurotrophic factors secreted from culture medium of NSCs, neurons, and sphere of neurons, and a total of $2 \mathrm{mg}$ total enriched protein combined with collagen scaffold were transplanted into completely transected T8 $\mathrm{SCI}$ in rat. BBB score analysis showed that the secreted neurotrophic factors from NSCs, neurons, and sphere of neurons would promote functional recovery of SCI to the same extent.

Injury to spinal cord leads to neural cell death and disruption of connection of brain and spinal cord below the lesion. NSCs-based therapy is a promising strategy for SCI treatment because they have capacity to differentiation into neurons, astrocytes, and oligodendrocytes, 
which could replenish the loss of functional cells and rebuild the disrupted neural connection in lesion sites. Nevertheless, SCI causes a series of pathological changes including vascular damage, ischemia, tissue edema, neuron interruption, inflammatory factors/cells aggregation, demyelination, glia scar and cystic cavity formation, forming an adverse niche for subsequent nerve regeneration[1-3, 16]. In addition, the released myelin-related proteins such as Nogo, tenascin, oligodendrocyte myelin glycoprotein (OMgp), and myelin based glycoprotein (MAG), which pose another obstacle for neural self-repair after SCI[7]. In our previous study, we found that crude myelin or myelin-related protein, Nogo-A protein could dramatically stump the neuronal differentiation of NSCs in vitro, which was reinforced by accumulating evidence[7, 17]. Thus, we put forward a novel therapy a novel strategy for SCI repair by transplanting in vitro differentiated neurons into the injured site, to avoiding the effect of adverse regenerative niche on neuronal differentiation inhibition of NSCs. In present study, we found the in vitro neuron differentiation of single-cell NSCs in cell density dependent manner and was hampered by crude myelin protein isolated from rat spinal cord, but intact NSCs sphere were capable to antagonize the neuronal inhibition posed by myelin protein. In rat acute transected SCI model, allogeneic NSCs sphere transplant did not achieved more newborn neurons in lesion site and preferable cell therapy associated functional improvement, compared with the transplant of single NSCs digested from sphere. This indicated the in vivo regeneration inhibitory niche is complex, among which myelin-associated proteins may do not play essential role. One study investigated the role of three major myelin-associated inhibitors, MAG, OMgp, and Nogo in injury-induced axonal growth by comprehensive genetic analysis[18]. In mice, deleting any one myelin-associated inhibitor increased the compensating sprouting of raphespinal serotonergic or corticospinal axons, but 
382

383

without associated behavioral improvement or a synergistic effect while deleting all three inhibitors. In addition, the regeneration of either axonal tract was not observed in triple-mutant mice after SCI. These results reveal MAG, OMgp, and Nogo do not play predominant role in the axon regeneration failure of spinal cord, while they are capable to modulate the sprouting of axons.

To avoid the effect of regeneration inhibitory microenvironment on grafted NSCs in injured spinal cord, we differentiated the enzymatically digested NSCs and NSCs sphere in vitro and then transplanted the mixtures of differentiated cells including neurons, astrocytes, undifferentiated NSCs combined with collagen scaffold into injured sites after SCI. We expected the high proportion of neurons among the differentiated cells had more powerful role in SCI recovery after transplant. Albeit each cell treatment markedly improved the locomotor recovery after SCI compared with alone collagen scaffold, unexpectedly there was no significant deference in behavioral improvement between the differentiated cell transplant and undifferentiated cell transplant. In addition, we also did not observe more newborn neurons (Tuj-1 positive neurons) in injured site in differentiated cell transplant. This result is probably caused by the adverse niche to neuron growth in injured site after SCI. The beforehand in vitro differentiated neurons derived NSCs are vulnerable to adverse niche at injured sites and difficultly survive after grafting due to their intolerance to such severe condition. The immune rejection between host and graft is another possible factor which cleans out the transplanted differentiated neurons in SCI. Cyclosporin A has a strong immunosuppressive effect by inhibiting the proliferation of $\mathrm{T}$ cells and $\mathrm{B}$ cell subsets. Although the immune suppressor Cyclosporin D was routinely administrated to rats throughout the test period, maybe it could not completely suppress the immune rejection of allogenic cells and 
lead to the death of most beforehand differentiated neurons or newborn neurons derived from grafted allogeneic NSCs.

$$
\text { The allogeneic differentiated neurons were difficult to survive at injured site, thus they }
$$
should have other mechanisms than replacement of lost neurons which contributes to functional recovery of SCI after graft. Each cell treatment group has similar functional improvement in SCI repair hints there is a same mechanism among all cell treatment groups. Transcriptome analysis revealed that there was an obvious diversity in enrichment of biochemical pathways among neuron and sphere of neuron, but without significant difference in mRNA and protein expressions of a variety of neurotrophic factors among NSCs, neuron, and sphere of neuron groups. NGF was the first discovered neurotrophic factor and thereafter a variety of neurotrophins were identified[19, 20]. The rodent and human CNS have high expressions of these neurotrophic factors and corresponding receptors, which play critical roles in the CNS development and homeostasis such as the synaptic plasticity, growth and stabilization of dendritic spines, glia and neurons' survival, long-term potentiation, and learning and memory[21]. NSCs have been shown to express high levels of various prominently studied neurotrophins such as NGF, BDNF, GDNF, IGF-1, and others, underlying the observed improvement in NSCs-based treatment of debilitating CNS disorders[22, 23]. Neurotrophic factors have been widely used to obtain significant success in improving behavioral and morphological outcomes when administered in or close to the lesion site after experimental SCI using various strategies such as cell-mediated delivery, osmotic minipumps, or supportive matrix[24]. We speculated that NSCs-based therapy in various forms improving locomotor function after SCI attributed to the secreting neurotrophic factors by various form cells such as undifferentiated or beforehand differentiated NSCs. Then we enriched the mixture of 
secreting neurotrophic factors from NSCs, neurons, and neurons of sphere freeze-drying corresponding culture medium. As representative neurotrophic factors, the BDNF and NGF concentrations enriched before and after were determined by ELISA, showing BDNF and NGF concentrations close to each other three groups. An equal dose of total enriched protein (2mg) from three kind culture mediums was administrated to rats with completely transected T8 SCI and a similar locomotor functional improvement was observed among three groups. These results demonstrated that allogeneic NSCs transplant differentiated before or after promoted functional recovery of locomotion after acute SCI predominantly by paracrine neurotrophic factors.

While the cell-based therapy for SCI recovery advances rapidly, achieving the neuronal replacement goal by stem cell transplant is far more complex than initially expected. First, the grafted stem cells immediately face the challenges of acute immune response and other adverse niche in injured site, leading to a difficult survival. Then the transplanted cells need to differentiate into the appropriate neuronal subtype with high fidelity. The most challenging is that these neurons derived from transplanted stem cells need to accomplish true neuronal replacement by projecting to the appropriate target neurons and forming appropriate synaptic connections, achieving the communication of nerve signals up and below the injured section of spinal cord. In addition, allogeneic NSCs transplant still face the immune rejection from host in the long run. Thus, further studies should aim at these aspects of challenges to achieve the concept of neuronal replacement by stem cell transplant in SCI recovery.

\section{Conclusion}

In this study, four kinds of NSCs and nerve growth factor were transplanted into completely transected T8 SCI in rats. The results show that allogeneic NSCs transplant promotes functional 
recovery of SCI predominantly by secreting neurotrophic factors, not direct neuronal replacement of differentiated neurons from transplanted cells.

\section{Abbreviations}

SCI: Spinal Cord Injury; NSCs: Neural Stem Cells; CNS: Central Nervous System; SD: Sprague- Dawley; SPF: Specific Pathogen Free; MBP: Myelin Basic Protein; GST: Glutathione S-transferase; FBS: Fetal Bovine Serum; PBS: Phosphate Buffered Saline; H\&E: Hematoxylin-eosin; BBB: Basso-Beattie-Bresnahan; PCA: Principal Component Analysis; PLS-DA: Partial least squares discrimination analysis; HCA: Hierarchical Cluster Analysis; GO: Gene Ontology; GAPDH: glyceraldehyde-3-phosphate dehydrogenase; ELISA: Enzyme-linked immunosorbent assay.

\section{Funding}

This research was supported by the National Key Research and Development Program of China (2017YFA0104304), the National Natural Science Foundation of China (NSFC) (81571213 (Bin Wang), 82070459(Bin Wang), 81800583 (Yuanyuan Xie) and 81901266 (Caixia Fan)), the Nanjing Medical Science and technique Development Foundation (QRX17006) (Bin Wang), and the Nanjing Medical Science and Innovation Platform (ZDX16005) (Bin Wang), Science and Technology Bureau of Yuzhong District ( 20140112 ) (Yanyan Cui)..

\section{Availability of data and materials}

The datasets used and/or analyzed during the current study are available from the corresponding author on reasonable request.

\section{Authors' contributions}

BW, SL and CxF designed the study. YyX, and LdW carried out the isolation and culture of NSCs. SL, CxF, YyX, LdW and YyC performed the other experiments and analyzed and formatted the data. SL wrote the first draft of the 
472 The study was approved by the Research Ethics Board of The Affiliated Drum Tower Hospital of Nanjing

Not applicable.

\section{Competing interests}

\section{References:}

479

1. Fujita, Y.; Yamashita, T., Axon growth inhibition by RhoA/ROCK in the central nervous system. Front Neurosci. 2014; 8:338.

482 2. Liu, K.; Tedeschi, A.; Park, K. K.; He, Z., Neuronal intrinsic mechanisms of axon regeneration. 483 Annu Rev Neurosci. 2011; 34:131-52.

484 3. Fawcett, J. W.; Schwab, M. E.; Montani, L.; Brazda, N.; Muller, H. W., Defeating inhibition of regeneration by scar and myelin components. Handb Clin Neurol. 2012; 109:503-22. 4. Lu, P.; Graham, L.; Wang, Y.; Wu, D.; Tuszynski, M., Promotion of survival and differentiation of neural stem cells with fibrin and growth factor cocktails after severe spinal cord injury. J Vis Exp. 2014; (89) : e50641. 5. Barnabe-Heider, F.; Goritz, C.; Sabelstrom, H.; Takebayashi, H.; Pfrieger, F. W.; Meletis, K.; Frisen, J., Origin of new glial cells in intact and injured adult spinal cord. Cell Stem Cell. 2010; 7 (4) :

491 470-82.

492 6. Meletis, K.; Barnabe-Heider, F.; Carlen, M.; Evergren, E.; Tomilin, N.; Shupliakov, O.; 493 Frisen, J., Spinal cord injury reveals multilineage differentiation of ependymal cells. PLoS Biol. 2008; 6 494 (7) : e182.

495 7. Wang, B.; Xiao, Z.; Chen, B.; Han, J.; Gao, Y.; Zhang, J.; Zhao, W.; Wang, X.; Dai, J., 496 Nogo-66 promotes the differentiation of neural progenitors into astroglial lineage cells through 497 mTOR-STAT3 pathway. PLoS One. 2008; 3 (3) : e1856.

498 8. Xie, F.; Zheng, B., White matter inhibitors in CNS axon regeneration failure. Exp Neurol. 2008; 209 $499 \quad(2): 302-12$.

500 9. Vroemen, M.; Aigner, L.; Winkler, J.; Weidner, N., Adult neural progenitor cell grafts survive 501 after acute spinal cord injury and integrate along axonal pathways. Eur J Neurosci. 2003; 18 (4) : 743-51.

503 10. Cao, Q. L.; Zhang, Y. P.; Howard, R. M.; Walters, W. M.; Tsoulfas, P.; Whittemore, S. R., 504 Pluripotent stem cells engrafted into the normal or lesioned adult rat spinal cord are restricted to a 505 glial lineage. Exp Neurol. 2001; 167 (1) : 48-58. 
11. Mothe, A. J.; Kulbatski, I.; Parr, A.; Mohareb, M.; Tator, C. H., Adult spinal cord stem/progenitor cells transplanted as neurospheres preferentially differentiate into oligodendrocytes in the adult rat spinal cord. Cell Transplant. 2008; 17 (7) : 735-51.

12. Ankeny, D. P.; Popovich, P. G., Mechanisms and implications of adaptive immune responses after traumatic spinal cord injury. Neuroscience. 2009; 158 (3) : 1112-21.

13. Stenudd, M.; Sabelstrom, H.; Frisen, J., Role of endogenous neural stem cells in spinal cord injury and repair. JAMA Neurol. 2015; 72 (2) : 235-7.

14. Zhu, T.; Tang, Q.; Gao, H.; Shen, Y.; Chen, L.; Zhu, J., Current status of cell-mediated regenerative therapies for human spinal cord injury. Neurosci Bull. 2014; 30 (4) : 671-82.

15. Norton, W. T.; Poduslo, S. E., Myelination in rat brain: method of myelin isolation. J Neurochem. 1973; 21 (4) : 749-57.

16. Liu, S.; Xie, Y. Y.; Wang, B., Role and prospects of regenerative biomaterials in the repair of spinal cord injury. Neural Regen Res. 2019; 14 (8) : 1352-1363.

17. Li, X.; Fu, Q. L.; Jing, X. L.; Liao, X. X.; Zeng, A. H.; Xiong, Y.; Liao, X. X., Myelin-associated glycoprotein inhibits the neuronal differentiation of neural progenitors. Neuroreport. 2009; 20 (7) : 708-12.

18. Lee, J. K.; Geoffroy, C. G.; Chan, A. F.; Tolentino, K. E.; Crawford, M. J.; Leal, M. A.; Kang, B.; Zheng, B., Assessing spinal axon regeneration and sprouting in Nogo-, MAG-, and OMgp-deficient mice. Neuron. 2010; 66 (5) : 663-70.

19. Levi-Montalcini, R.; Hamburger, V., Selective growth stimulating effects of mouse sarcoma on the sensory and sympathetic nervous system of the chick embryo. J Exp Zool. 1951; 116 (2) : 321-61.

20. Nagahara, A. H.; Tuszynski, M. H., Potential therapeutic uses of BDNF in neurological and psychiatric disorders. Nat Rev Drug Discov. 2011; 10 (3) : 209-19.

21. Vicario-Abejon, C.; Owens, D.; McKay, R.; Segal, M., Role of neurotrophins in central synapse formation and stabilization. Nat Rev Neurosci. 2002; 3 (12) : 965-74.

22. Kamei, N.; Tanaka, N.; Oishi, Y.; Hamasaki, T.; Nakanishi, K.; Sakai, N.; Ochi, M., BDNF, NT-3, and NGF released from transplanted neural progenitor cells promote corticospinal axon growth in organotypic cocultures. Spine (Phila Pa 1976) . 2007; 32 (12) : 1272-8.

23. Marsh, S. E.; Blurton-Jones, M., Neural stem cell therapy for neurodegenerative disorders: The role of neurotrophic support. Neurochem Int. 2017; 106: 94-100.

24. Hodgetts, S. I.; Harvey, A. R., Neurotrophic Factors Used to Treat Spinal Cord Injury. Vitam Horm. 2017; 104 :405-457.

\section{Figure legends:}

Figure 1 NSCs sphere had a high neuronal differentiation potential, antagonizing the neuronal differentiation of myelin protein. A: The immunofluorescence of GFAP and Tuj-1 
antibodies showed the neuronal differentiation of NSCs in a density-dependent manner. B: The qualified data of Tuj-1 positive staining showed the NSC sphere had a maximal neuronal differentiation potential. C: The hierarchical scan of immunostaining showed the majority of differentiated cells were GFAP positive astrocytes after single-cell NSCs differentiated. D: The Tuj-1 positive neurons with long processes connected with each other were predominant after NSC sphere differentiated. E-F: Myelin could inhibit the neuronal differentiation of single-cell NSCs, without effect on intact NSC sphere.

Figure 2 All cell combined with collagen material groups had beneficial motor functional recovery in completely transected SCI model. A: The schematic diagram of experiments (Control group: without any treatment, Material group: collagen scaffold transplant, NSCs group: collagen scaffold plus primary enzyme digested NSCs, Sphere of NSCs group: collagen scaffold plus NSC sphere, Neurons group: collagen scaffold plus differentiated cells derived from enzyme digested NSCs, Sphere of neurons group: collagen scaffold plus differentiated cells derived from NSC sphere). B: All groups with cells combined with collagen materials had similar BBB scores at indicated time points. C: After 8 weeks post-surgery, H\&E staining showed a less tissue remodeling and huge cavity in control group and material treatment had a better tissue remodeling and smaller cavity, but all cell treatment groups had a similar outcome with a good tissue remodeling in injured sites.

Figure 3 The immune staining of Tuj-1 positive neurons. A: The panoramic scanning of the horizontal sections of spinal cord. Results showed Tuj-1 staining was heavily positive outside of 
injured site, but was clear-cut within damaged lesion. Scale bar $=1 \mathrm{~mm}$. B: In high-magnification of injured area, the positive of newborn neurons (Tuj-1+ cells) were sparse in control and material groups, much less than those in other four groups with different cell transplant. Scale bar=100 $\mu \mathrm{m}$. C: Quantification of Tuj-1 staining in injured spinal cord. Overall, the percentages of Tuj-1+ neurons in groups with cell treatment were much higher than that in in control and material groups. ( $\mathrm{a}$ and $\mathrm{b}: \mathrm{p}<0.05$ and 0.01 compared to control group, respectively; $\mathrm{c}$ : $\mathrm{p}<0.01$ compared to material group, $\mathrm{d}: \mathrm{p}<0.001$ compared to control and material groups).

Figure 4 The transcriptome analysis. A: The heatmap showed the differentially expressed genes between neuron and sphere of neuron groups. Each column represents one cell sample and each row represents one gene. B: KEGG analysis identified significant enrichment of biochemical pathways among neuron and sphere of neuron groups and showed that neuron projection development related processes were mainly enriched in neuron samples, and cell adhesion and motility, proliferation processes were mainly enriched in sphere of neuron samples.

Figure 5 The heatmap focused on mRNA expressions of neurotrophic factors among NSC, neuron, and sphere of neuron groups. was performed to determine the protein expressions of BDNF and NGF in medium among NSCs, neuron, and sphere of neuron groups. 
589 Figure 7 Complex of neurotrophic factors promoting locomotor functional recovery. A: The

590 concentrations of both NGF and BDNF in enriched medium were about 7-times higher than that in

591 basal medium. B: A total of $2 \mathrm{mg}$ total enriched protein secreted from NSCs, neurons, and sphere

592 of neurons respectively combined with collagen scaffold and transplanted into completely

593 transected SCI model. The BBB scores were similar among three groups.

594 


\section{Figures}

A

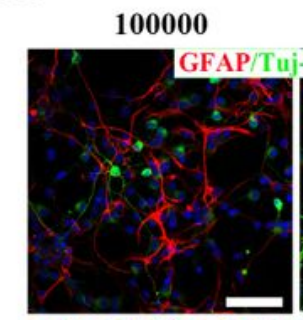

B
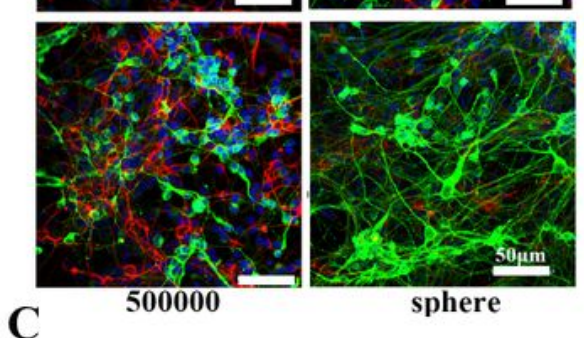

C

sphere

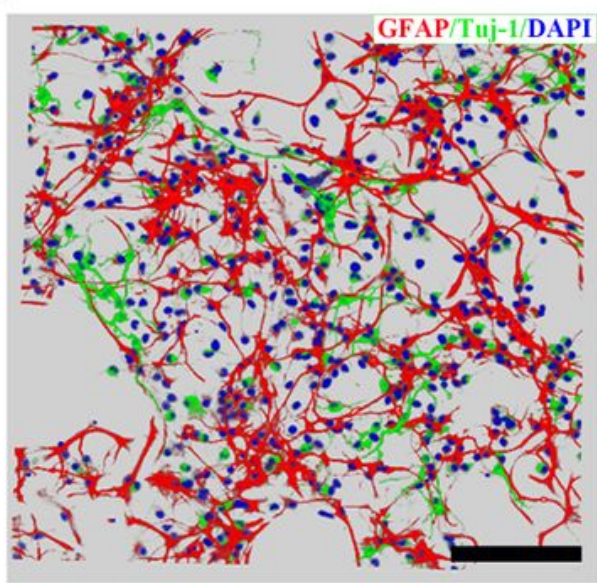

E

BSA

Myelin
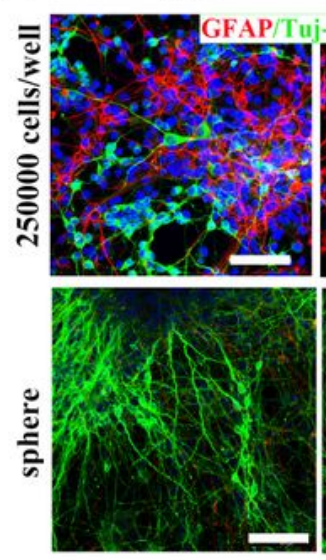

250000 cells/well
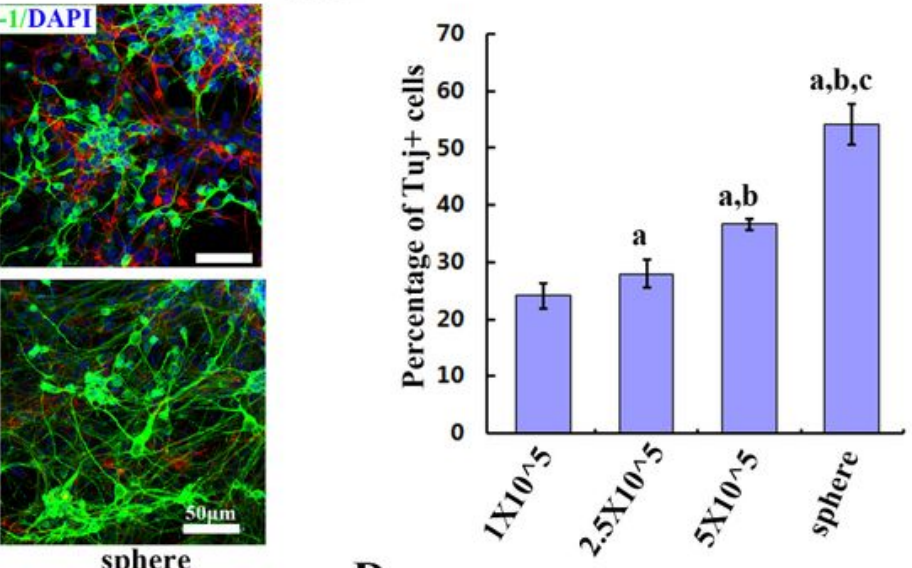

D

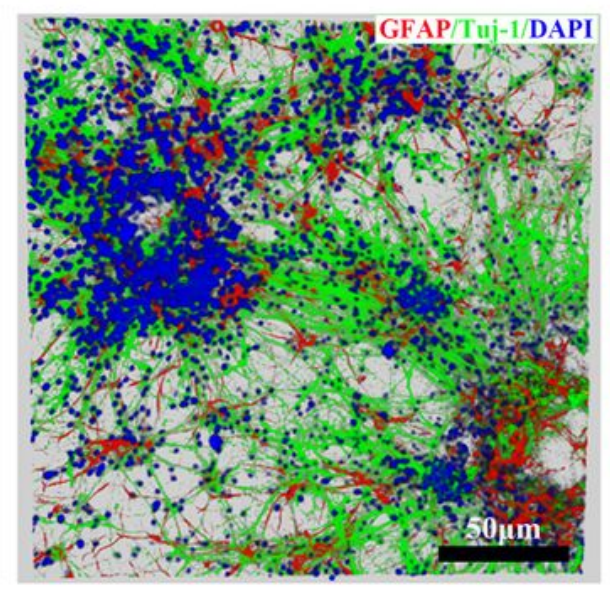

F
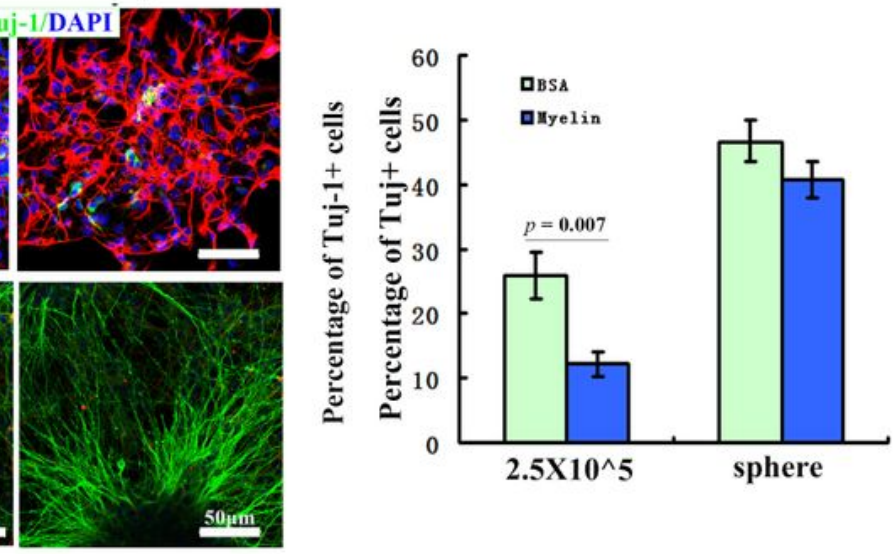

Figure 1

NSCs sphere had a high neuronal differentiation potential, antagonizing the neuronal differentiation of myelin protein. A: The immunofluorescence of GFAP and Tuj-1. antibodies showed the neuronal differentiation of NSCs in a density-dependent manner. B: The qualified data of Tuj-1 positive staining 
showed the NSC sphere had a maximal neuronal differentiation potential. C: The hierarchical scan of immunostaining showed the majority of differentiated cells were GFAP positive astrocytes after singlecell NSCs differentiated. D: The Tuj-1 positive neurons with long processes connected with each other were predominant after NSC sphere differentiated. E-F: Myelin could inhibit the neuronal differentiation of single-cell NSCs, without effect on intact NSC sphere.

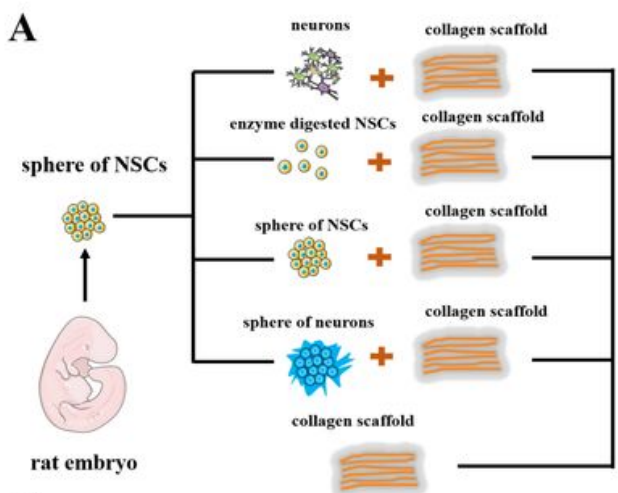

C

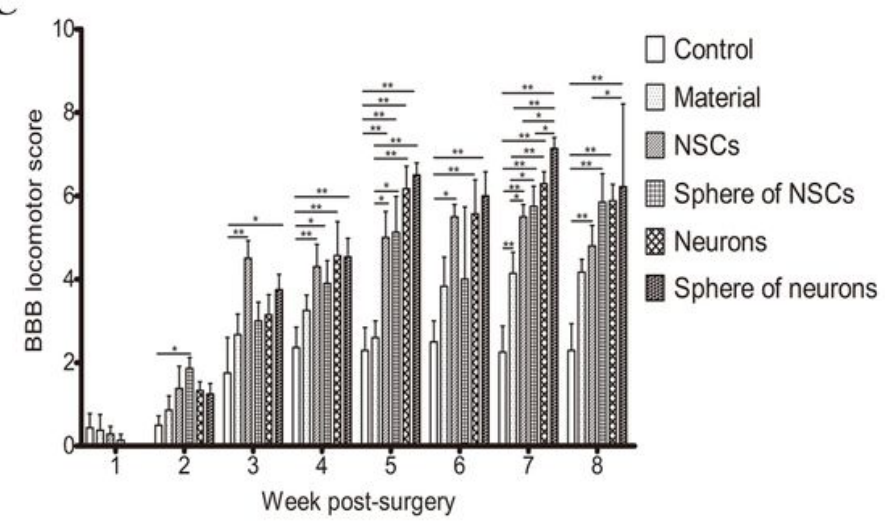

B
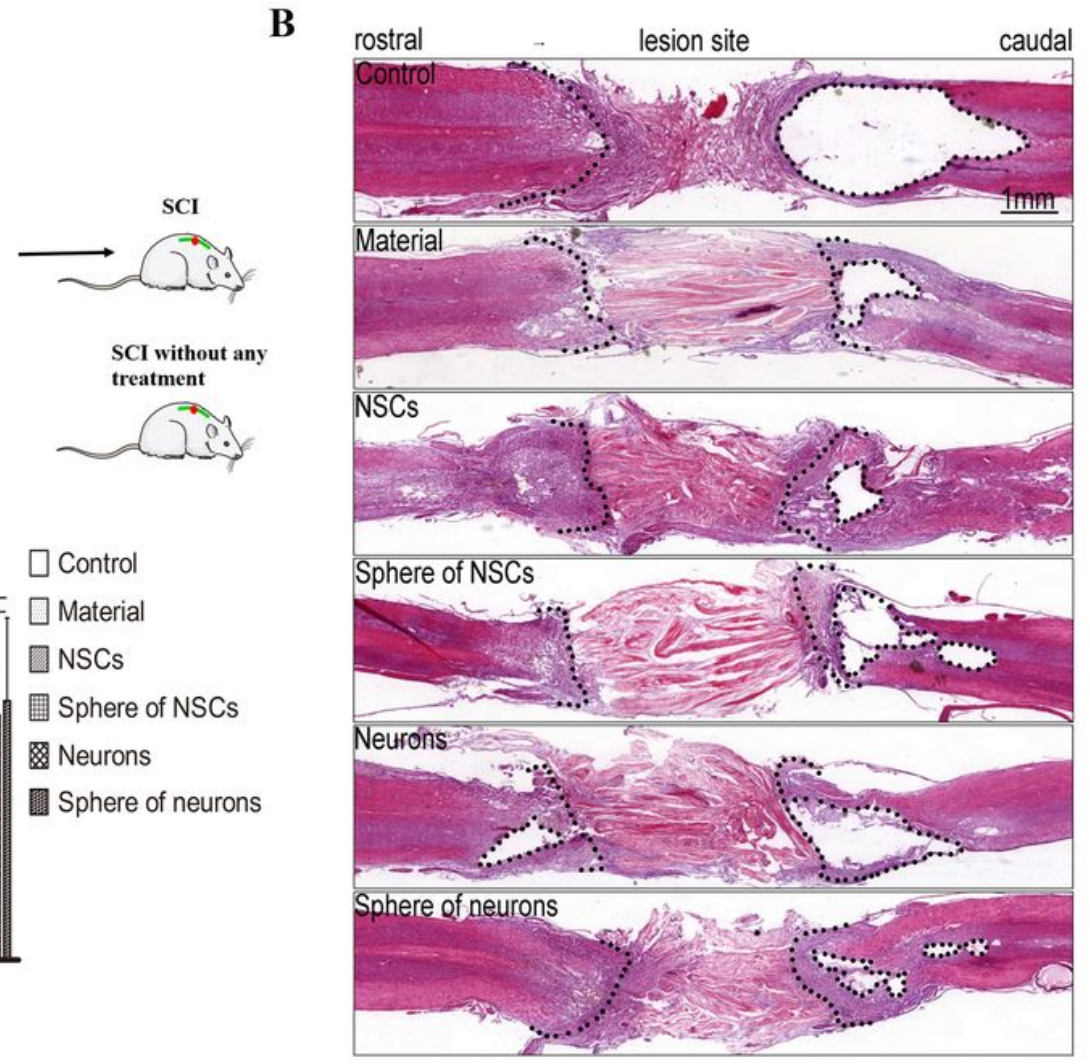

\section{Figure 2}

All cell combined with collagen material groups had beneficial motor functional recovery in completely transected SCI model. A: The schematic diagram of experiments (Control group: without any treatment, Material group: collagen scaffold transplant, NSCs group: collagen scaffold plus primary enzyme digested NSCs, Sphere of NSCs group: collagen scaffold plus NSC sphere, Neurons group: collagen scaffold plus differentiated cells derived from enzyme digested NSCs, Sphere of neurons group: collagen scaffold plus differentiated cells derived from NSC sphere). B: All groups with cells combined with collagen materials had similar BBB scores at indicated time points. C: After 8 weeks post-surgery, H\&E staining showed a less tissue remodeling and huge cavity in control group and material treatment had a 
better tissue remodeling and smaller cavity, but all cell treatment groups had a similar outcome with a good tissue remodeling in injured sites.

A
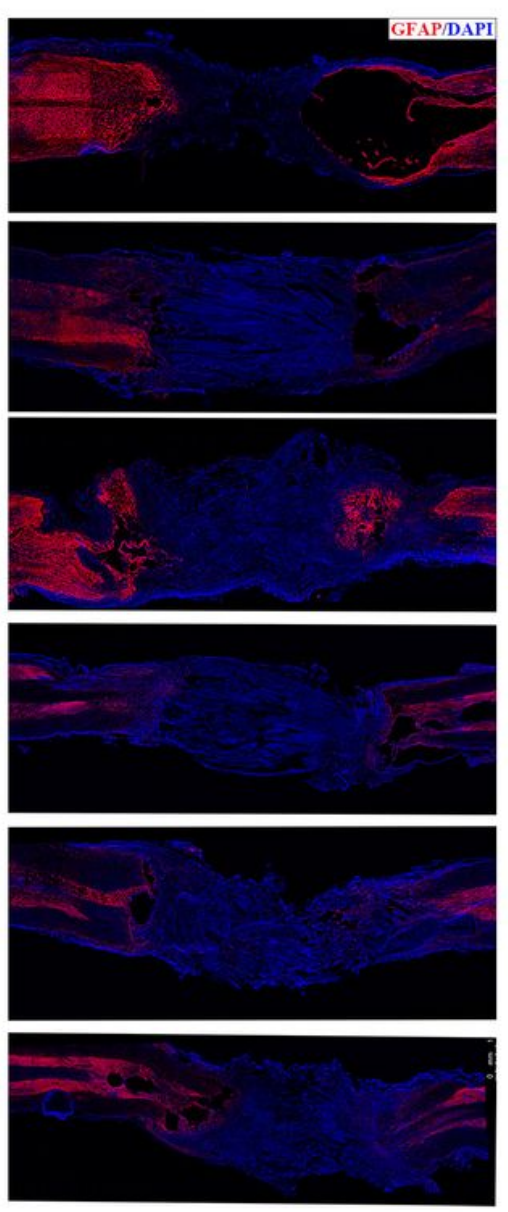

B
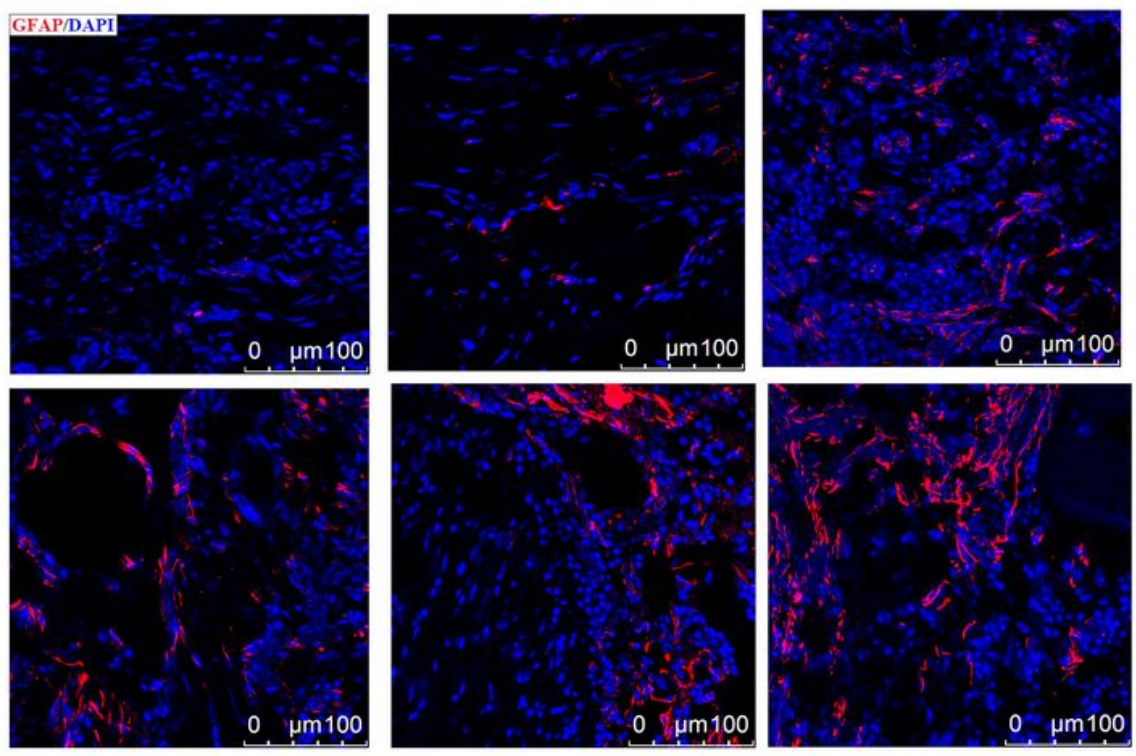

C

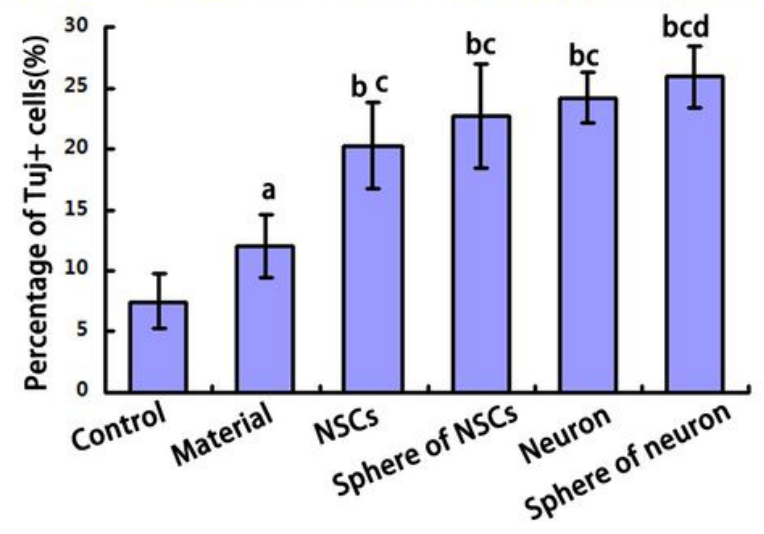

\section{Figure 3}

The immune staining of Tuj-1 positive neurons. A: The panoramic scanning of the horizontal sections of spinal cord. Results showed Tuj-1 staining was heavily positive outside of injured site, but was clear-cut within damaged lesion. Scale bar $=1 \mathrm{~mm}$. B: In high-magnification of injured area, the positive of newborn neurons (Tuj-1+ cells) were sparse in control and material groups, much less than those in other 
four groups with different cell transplant. Scale bar $=100 \mu \mathrm{m}$. C: Quantification of Tuj-1 staining in injured spinal cord. Overall, the percentages of Tuj-1+ neurons in groups with cell treatment were much higher than that in in control and material groups. ( $a$ and $b: p<0.05$ and 0.01 compared to control group, respectively; $\mathrm{c}$ : $<0.01$ compared to material group, $d$ : $p<0.001$ compared to control and material groups).

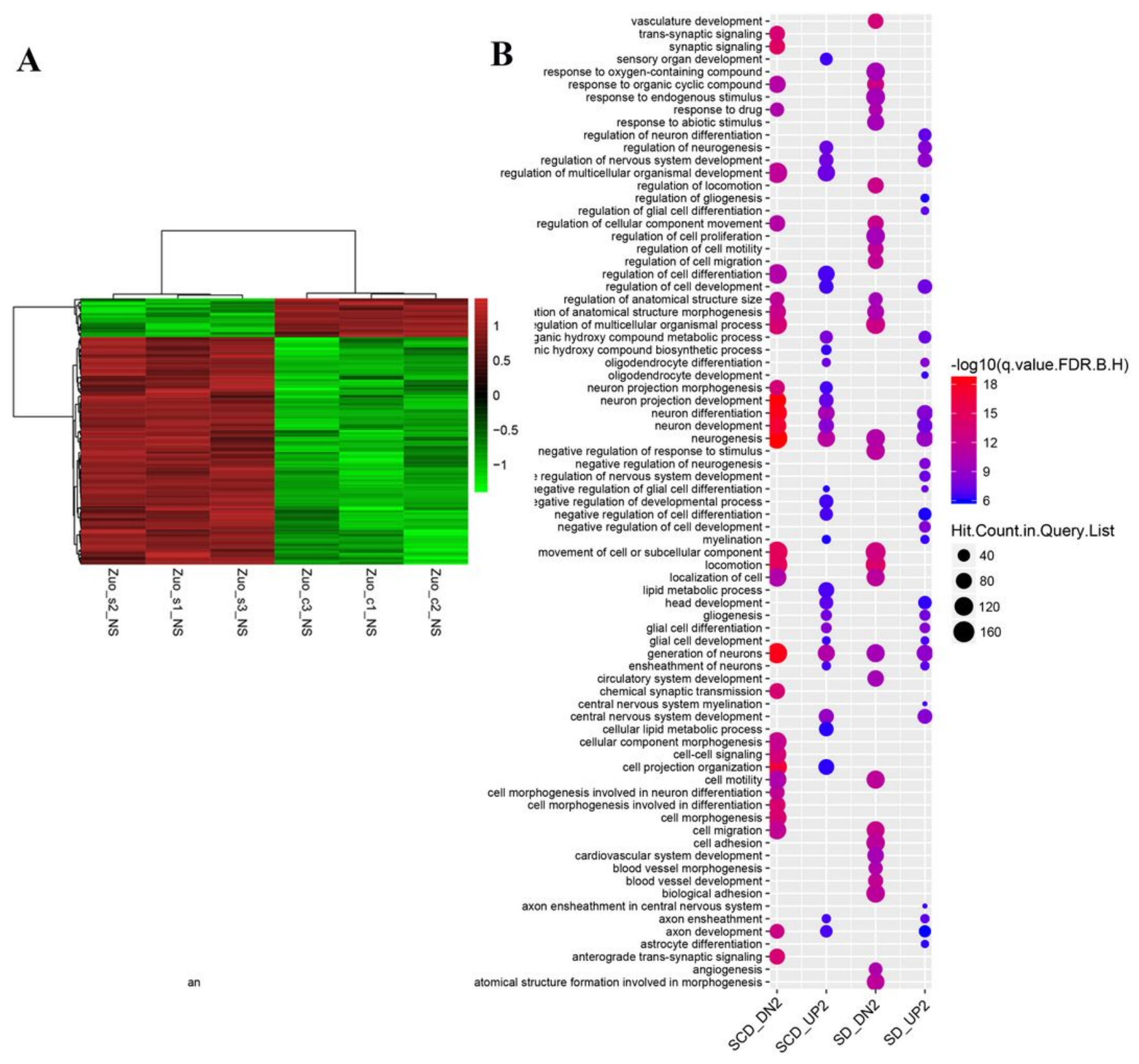

\section{Figure 4}

The transcriptome analysis. A: The heatmap showed the differentially expressed genes between neuron and sphere of neuron groups. Each column represents one cell sample and each row represents one gene. B: KEGG analysis identified significant enrichment of biochemical pathways among neuron and sphere of neuron groups and showed that neuron projection development related processes were mainly enriched in neuron samples, and cell adhesion and motility, proliferation processes were mainly enriched in sphere of neuron samples. 


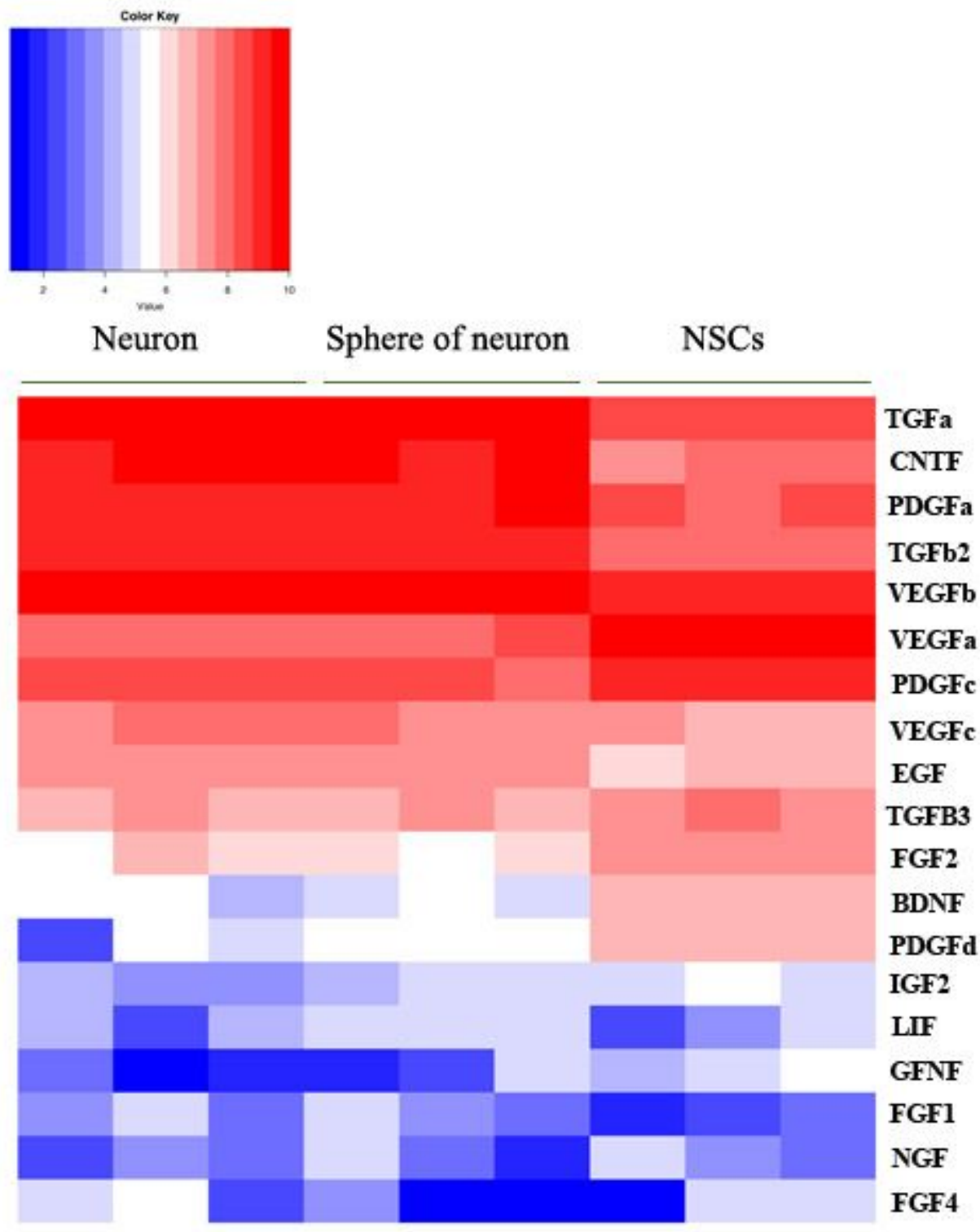

Figure 5

The heatmap focused on mRNA expressions of neurotrophic factors among NSC, neuron, and sphere of neuron groups. 
A

VEGF

BDNF

GDNF

TGF $\alpha$
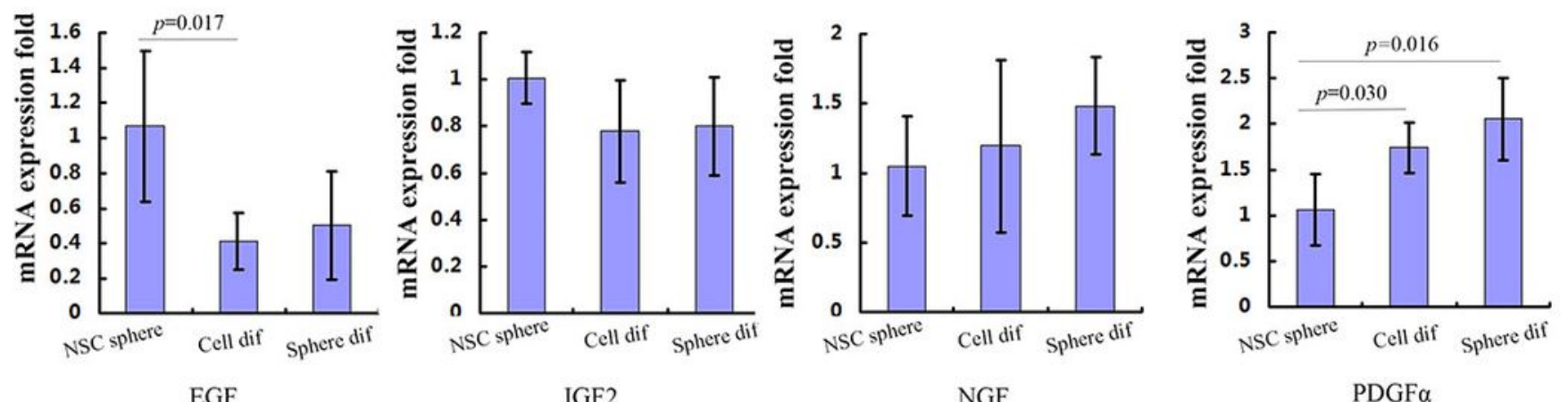

IGF2

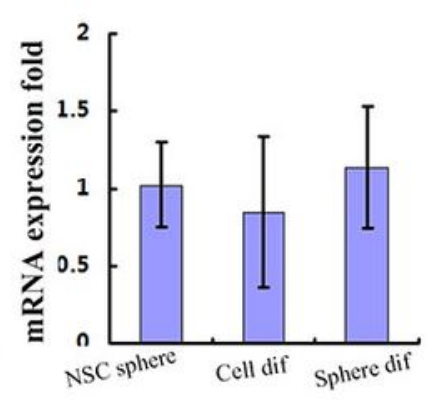

PDGF $\alpha$
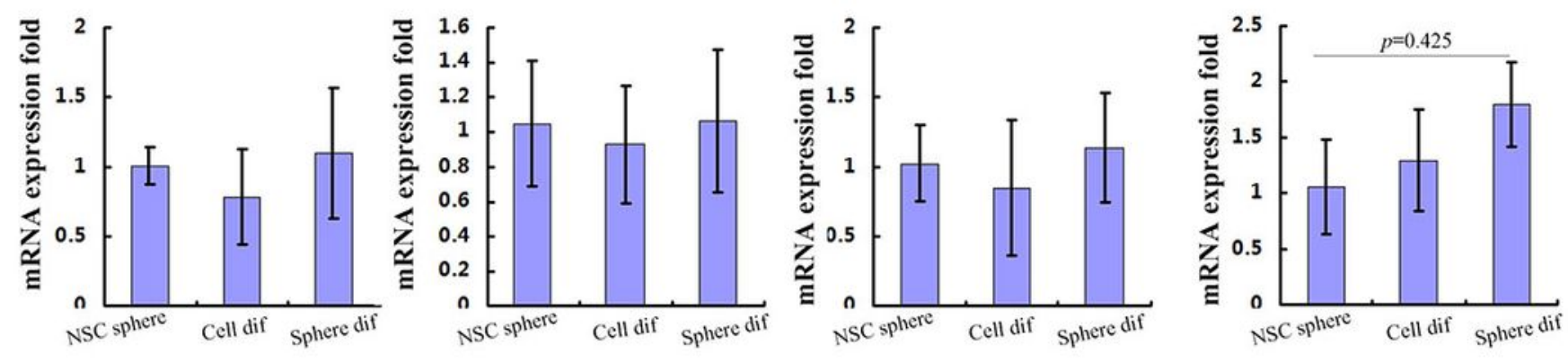

bFGF

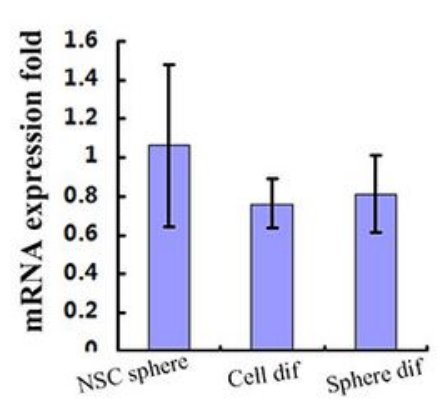

LIF
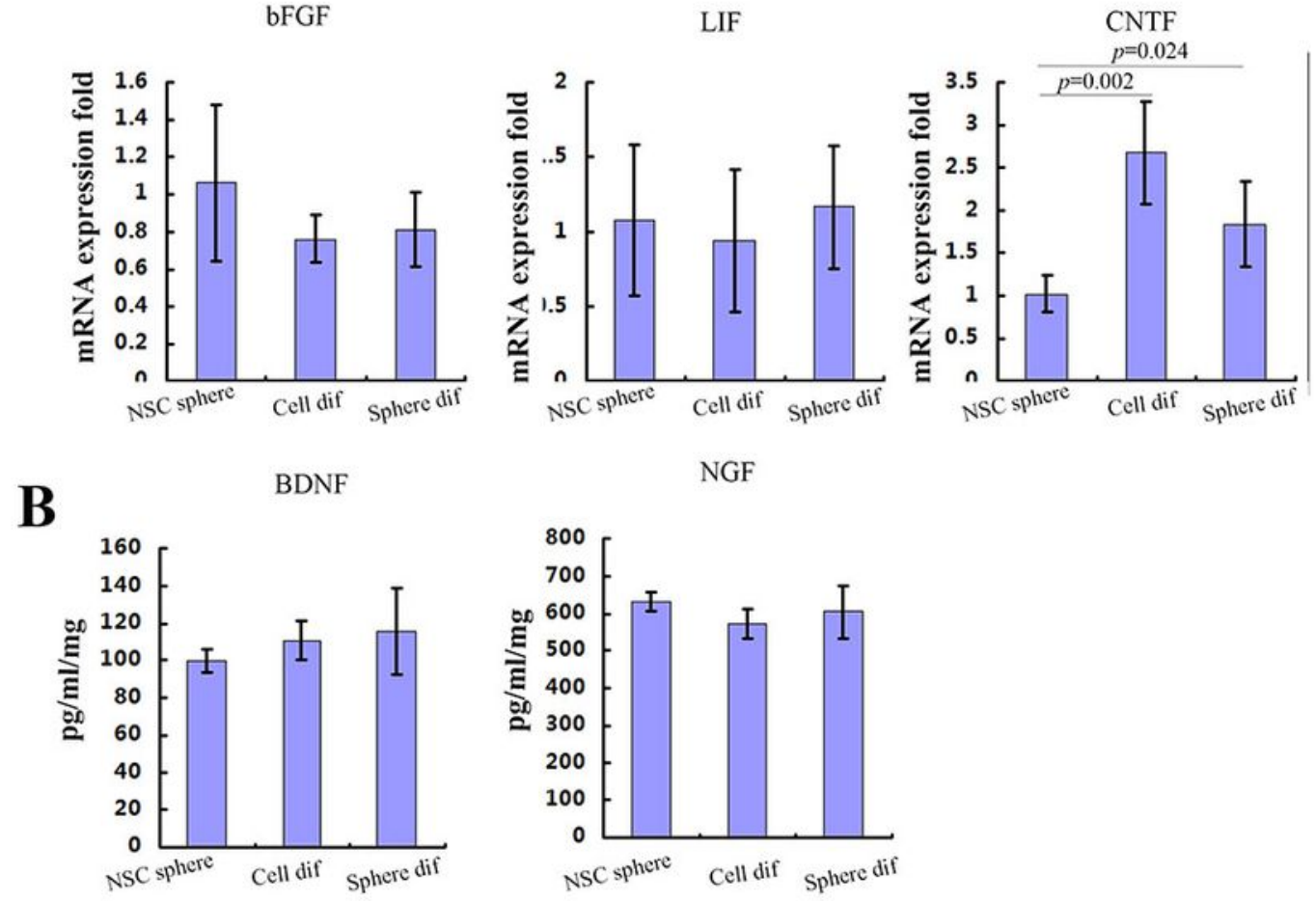

\section{Figure 6}

Validating the neurotrophic factors among NSCs, neuron, and sphere of neuron groups. A: The mRNA expressions of neurotrophic factors were analyzed by qPCR. B: ELISA was performed to determine the protein expressions of BDNF and NGF in medium among NSCs, neuron, and sphere of neuron groups. 
A
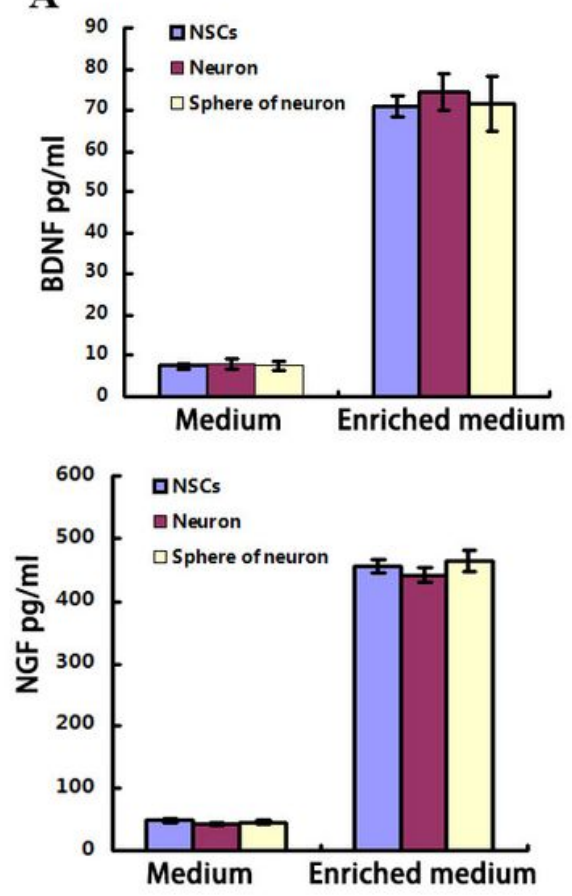

B

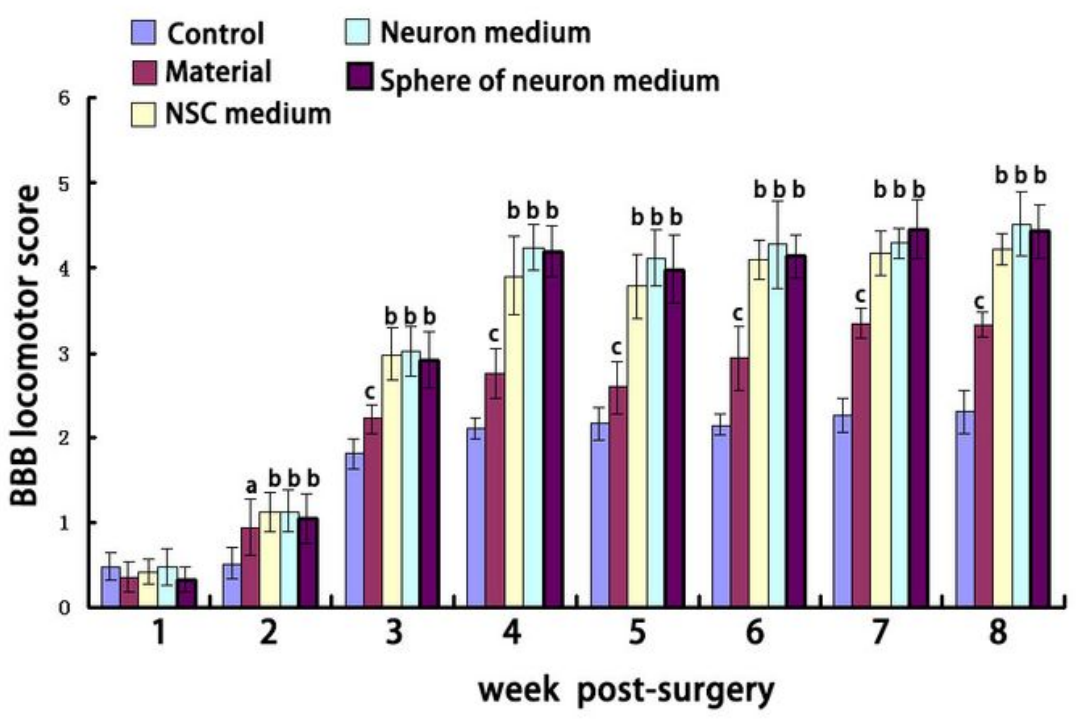

Figure 7

Complex of neurotrophic factors promoting locomotor functional recovery. A: The concentrations of both NGF and BDNF in enriched medium were about 7-times higher than that in basal medium. B: A total of 2 mg total enriched protein secreted from NSCs, neurons, and sphere of neurons respectively combined with collagen scaffold and transplanted into completely transected SCI model. The BBB scores were similar among three groups. 